\title{
BMJ Open Peripherally Inserted Central catheter iNnovation to reduce Infections and Clots (the PICNIC trial): a randomised controlled trial protocol
}

\author{
Amanda J Ullman (D) , ${ }^{1,2,3,4}$ Deanne August, ${ }^{1,2,4}$ Tricia Kleidon, ${ }^{1,2,3}$ Rachel Walker, ${ }^{2,5}$ \\ Nicole M Marsh, ${ }^{1,2,4}$ Andrew Bulmer, ${ }^{2}$ Benjamin Pearch, ${ }^{5}$ Naomi Runnegar, ${ }^{5,6}$ \\ Jessica A Schults (D) , 1,3,4 Joanne Leema, ${ }^{2,5}$ Paul Lee-Archer, ${ }^{3,6}$ Cathy Biles, ${ }^{5}$ \\ Katrina Southam, ${ }^{4}$ Victoria Gibson, ${ }^{1,2,3}$ Joshua Byrnes, ${ }^{2}$ Robert S Ware (1) , \\ Vineet Chopra, ${ }^{2,7}$ Alan Coulthard, ${ }^{4,6}$ Peter Mollee, ${ }^{5,6}$ Claire M Rickard, ,,2,4 \\ Patrick N A Harris ${ }^{4,6}$
}

To cite: Ullman AJ, August D, Kleidon T, et al. Peripherally Inserted Central catheter iNnovation to reduce Infections and Clots (the PICNIC trial): a randomised controlled trial protocol. BMJ Open 2021;11:e042475. doi:10.1136/ bmjopen-2020-042475

- Prepublication history and additional supplemental material for this paper are available online. To view these files, please visit the journal online (http://dx.doi.org/10.1136/ bmjopen-2020-042475)

Received 06 July 2020 Revised 11 January 2021 Accepted 31 March 2021
Check for updates

(C) Author(s) (or their employer(s)) 2021. Re-use permitted under CC BY-NC. No commercial re-use. See rights and permissions. Published by BMJ.

For numbered affiliations see end of article.

Correspondence to Professor Amanda J UIIman; a.ullman@uq.edu.au

\section{ABSTRACT}

Introduction Peripherally inserted central catheters (PICCS) are vital for the delivery of medical therapies, but up to $30 \%$ of PICCs are associated with complications such as deep vein thrombosis or infection. The integration of antimicrobial and hydrophobic catheter materials, and pressure-activated valves, into polyurethane PICCs are innovations designed to prevent infective and/or thrombotic complications.

Methods and analysis A multicentre, parallel group, superiority randomised controlled trial with two experimental arms ((1) hydrophobic PICC (with pressureactivated valve); (2) chlorhexidine gluconate-impregnated PICC (with external clamp)) and one control group ((3) conventional polyurethane PICC (with external clamp)). Recruitment of 1098 adult and paediatric patients will take place over 2 years at three tertiary-referral hospitals in Queensland, Australia. Patients are eligible for inclusion if their PICC is to be inserted for medical treatment, with a vascular size sufficient to support a 4-Fr PICC or larger, and with informed consent. The primary outcome is PICC failure, a composite of thrombotic (venous thrombosis, breakage and occlusion) and infective complications (PICC-associated bloodstream infection and local infection). Secondary outcomes include: allcause PICC complication; thrombotic complications; infective complications; adverse events (local or systemic reaction); PICC dwell time; patient/parent satisfaction; and healthcare costs. Differences between both intervention groups and the control group will be compared using Cox proportional hazards regression. Effect estimates will be presented as $\mathrm{HRs}$ with corresponding $95 \% \mathrm{Cl}$.

Ethics and dissemination Ethical approval from Queensland Health (HREC/QCHQ/48682) and Griffith University (Ref. No. 2019/094). Results will be published. Trial registration number ACTRN12619000022167.

\section{INTRODUCTION}

Peripherally inserted central catheters (PICCs) are inserted via deep peripheral

\section{Strengths and limitations of this study}

- This is an adequately powered, multicentre, randomised controlled trial in adults and children to provide much-needed evidence for the clinical effectiveness and cost-effectiveness of new peripherally inserted central catheters (PICCs) that feature updated designs and materials.

- Allocation will not be concealed to clinical staff, since they must insert, access and monitor PICCs; however, blinded infectious disease physicians and interventional radiologists will assign infectious and thrombotic outcomes according to standardised definitions, and data analysis will be performed by a blinded statistician.

- The trial is from a single region that may limit the generalisability of the findings, and data for children will be limited due to the unavailability of smaller sizes of some PICCs.

veins, predominantly of the upper arm, into the central vasculature. ${ }^{1}$ PICCs are vital for many acute and chronic diagnoses requiring prolonged and/or frequent therapy, vesicant medications, irritant $\mathrm{pH}$ and osmolality infusions, for patients with limited veins in both outpatient and inpatient settings. ${ }^{2}$ The last 40 years have seen rapid acceleration in PICC use, due to their relative safety, ease of use and costeffectiveness compared with more invasive centrally inserted devices. However, it is now established that PICC-related complications are universally common, with up to $30 \%$ of PICCs failing prior to completion of treatment due to thrombotic (eg, deep vein thrombosis (DVT)) and infective complications (eg, catheter-associated bloodstream 
infection (CABSI) including those caused by Staphylococcus aureus) ${ }^{3-5}$ Innovations that prevent these complications are necessary to reduce healthcare-associated harm for vulnerable patients.

\section{PICC materials}

Integrating antithrombotic and anti-infective technologies into the PICG materials are potential innovations to prevent complications. Several PICC materials have been developed since their inception in the $1970 \mathrm{~s}^{6}$; however, the majority of PICCs currently in use are made of relatively stiff, third-generation uncoated polyurethane that softens with body temperature and withstands pressures of $>300$ pounds per square inch. ${ }^{6}$

Material technologies introduce antithrombotic and anti-infective properties in PICCs, with two major products entering the market over the last $5-10$ years. The BioFlo PICC from AngioDynamics Inc (Queensbury, New York, USA) has hydrophobic (water repelling) properties, which has been achieved by the addition of 'Endexo' (a surface-modifying macromolecule based on the incorporation of fluorine atoms) during the moulding manufacture process. The fluorinated PICC material inhibits platelet adhesion, suppresses protein procoagulant conformation and potentially reduces thrombus formation in medical devices (based on platelet counts in manufacturer completed in vitro studies only). ${ }^{67}$ Our pilot randomised controlled trial (RCT) in a paediatric population $(n=150)$ demonstrated the feasibility and potential for the hydrophobic BioFlo PICC to reduce PICC thrombotic and infective complications, but the study was not powered for clinical effectiveness (PICG failure: standard polyurethane $22 \%(16 / 72)$ ) vs BioFlo $11 \%$ (8/72): risk ratio (RR) $0.50 ; 95 \%$ CI 0.23 to $1.09 ; \mathrm{p}=0.07){ }^{8}$

Anti-infective properties impregnated into PICCs include antibiotics (eg, minocycline-rifampicin) and antiseptics (eg, chlorhexidine gluconate (CHG)). These technologies have been well studied in other central venous catheter types, with a Cochrane review ${ }^{9}$ concluding there was high-quality evidence that antimicrobial (non-PICC) central venous catheters significantly reduced CABSI risk (RR $0.62 ; 95 \%$ CI 0.52 to 0.74 , $\mathrm{n}=10405,42$ RCTs). Building on this indication, Teleflex Inc (Wayne, Pennsylvania, USA) developed the CHGimpregnated Arrowg+ard Blue Advance PICC. CHG is widely used in healthcare, due to its broad spectrum of germicidal activity against most CABSI-causing pathogens, especially $S$. aureus. ${ }^{10}$ Some manufacturer-funded, laboratory studies also suggest CHG coating has a role in the prevention of thrombosis development, with in vivo ovine models demonstrating a significant reduction in fibrin sheath development after 30 days in situ, in comparison with uncoated catheters (median $0.05 \mathrm{~g}$ (CHG) vs $0.7 \mathrm{~g}$ (uncoated) ). ${ }^{11}$ However, the effectiveness of CHG-coated PICCs to prevent thrombotic and infective complications has not been formally tested in clinical trials in humans.

\section{PICC designs}

PICC design evolution has primarily focused on the integration of internal valves, replacing external clamps, with the aim of preventing thrombotic occlusion by reducing blood reflux. ${ }^{6}$ External clamps prevent gravity and pressure-related backflow of blood into and out of the PICC when not in use. Modern pressure-activated valves, such as those incorporated in the BioFlo PICC from AngioDynamics Inc (Queensbury, New York, USA), negate the need for external, clinician-initiated clamping during periods of disuse. The valves are incorporated at either the distal or proximal portion of the PICC and open with injection and aspiration pressure but remain closed during minor pressure fluctuations. ${ }^{12}$ Despite the integration of valves into many PICCs, data regarding their performance in preventing complications in clinical trials are minimal. Only one RCT $(n=180)$ of the pressure activated valve versus older PICC valve technologies has been published, and this RCT was stopped early due to safety concerns with an older valve (Becton Dickinson - Bard, Murray Hill, New Jersey, USA). ${ }^{12}$

The ideal PICC would be: (A) soft and flexible to reduce procedural risks (including ease of insertion, patient discomfort and vessel irritation); (B) structurally strong to withstand pressure during rapid injection; $(\mathrm{C})$ prevent microbial colonisation and adherence of blood components to reduce CABSI and DVT; and (D) cost-effective. Little research has been undertaken examining the safety and effectiveness of either hydrophobic or CHG PICCs, with or without valves, to meet these criteria, prevent thrombotic and infection complications and promote PICC function. The aim of this study is to compare two novel technologies (hydrophobic and CHG PICCs) with standard care (conventional polyurethane PICCs), to identify clinically effective and cost-effective methods to reduce the incidence of PICC failure, due to thrombotic and infectious complications.

\section{METHODS AND ANALYSIS}

This multicentre, parallel group, open, superiority, randomised controlled trial will compare the clinical effectiveness and cost-effectiveness in adult and paediatric patients of the:

1. Hydrophobic PICC: with pressure activated valves; BioFlo.

2. CHG-impregnated PICC: with external clamps; Arrowg+ard Blue Advance.

3. Conventional polyurethane PICCs: pressure injectable polyurethane with external clamps.

Embedded in the RCT are additional microbiological and thrombogenic subgroup studies to determine the effect of these interventions on bacterial colonisation, PICC-associated infection and CHG resistance, as well as assessing haematological, coagulation profiles and platelet activation. 


\section{Hypotheses}

Primary

1. Patients with either (1) hydrophobic or (2) CHG PICCs will have significantly fewer device failures (composite outcome of thrombosis, infection, occlusion and breakage) compared with patients receiving (3) usual care (conventional polyurethane PICCs).

\section{Secondary}

1. Patients with the (1) hydrophobic and (2) CHG PICCs will have significantly longer complication-free device life compared with patients receiving (3) usual care (conventional polyurethane PICCs).

2. Patients with the (1) hydrophobic PICCs will have significantly lower rates of thrombotic complications (composite of thrombosis, occlusion and breakage), compared with patients receiving (3) usual care (conventional polyurethane PICCs).

3. Patients with (2) CHG PICCs will have significantly lower rates of PICC associated infections, than patients receiving (3) usual care (conventional polyurethane PICCs).

4. There will be significant cost savings to the Australian health system when using (1) hydrophobic or (2) CHG PICC, compared with patients receiving (3) usual care (conventional polyurethane PICCs).

\section{Study setting and sample}

Adult and paediatric patients are being recruited at three tertiary referral hospitals in South East Queensland, Australia (Royal Brisbane and Women's Hospital (RBWH); Princess Alexandra Hospital (PAH); and Queensland Children's Hospital $(\mathrm{QCH})$ ), aiming for recruitment over 2 years. Sites have phased recruitment commencement (QCH 8 November 2019; PAH 20 January 2020 and RBWH 1 June 2020), with delayed commencement at RBWH due to COVID-19. Patients requiring a PICC are being recruited via departments of medical imaging and vascular access services from cancer care, medical,

\begin{tabular}{ll}
\hline Table 1 Inclusion and exclusion criteria \\
\hline Inclusion criteria & Exclusion criteria \\
\hline PICC to be inserted for & Previous enrolment in the current \\
medical treatment. & study. \\
Informed consent. & Current catheter-related \\
Vascular size sufficient & bloodstream infection. \\
to support 4-Fr PICC & Thrombosis in vein where PICC \\
or larger. & is to be inserted. \\
& Non-English speaking without an \\
& interpreter. \\
& Known sensitivity to study \\
& products (including CHG). \\
& Added since trial \\
& commencement: admitted for \\
& COVID-19, or to a designated \\
& COVID-19 unit/facility.
\end{tabular}

CHG, chlorhexidine gluconate; Fr, French; PICC, peripherally inserted central catheter. surgical and intensive care areas. Patients who meet all inclusion and no exclusion criteria (see table 1) are eligible to participate in the study once.

\section{Outcome measures and definitions \\ Primary outcome}

The primary outcome is PICC failure, a composite of thrombotic (venous thrombosis, breakage and occlusion) and infective complications (PICC-associated bloodstream infection and local infection) (all defined further) severe enough to cause cessation of PICC function prior to therapy completion. ${ }^{513}$

- Venous thrombosis: ultrasound/venographic/image confirmed thrombosis occurring within the same vessel as the PICC within 1 week of PICC removal, in a symptomatic patient (pain and swelling), ${ }^{14}$ confirmed by blinded radiologist.

- Breakage: split in PICC material with leakage or radiographic evidence of extravasation/infiltration into tissue, in a PICC flushed to clear thrombosis and/or occlusion. ${ }^{15}$

- Occlusion: complete: $\geq 1$ lumens cannot be flushed or aspirated, or resolved post-thrombolytic dwell ${ }^{16}$ and partial: decreased ability of $\geq 1$ lumens to either infuse blood or fluid and/or withdraw blood or fluid from $\geq 1$ lumens despite the use of thrombolytic. ${ }^{17}$

- PICC-associated bloodstream infection (BSI): a laboratory confirmed BSI where an eligible BSI organism is identified with PICG in place for $>2$ consecutive calendar days on the day of the BSI (day of PICC placement being day 1) and the PICC in place on the date of the event or the day before (see CDC Device-associated Module BSI for full criteria), ${ }^{18}$ confirmed by a blinded infectious disease specialist.

- Local infection: clinical signs of local venous infection at the PICC insertion site (eg, purulent drainage), confirmed with a positive ( $>15 \mathrm{cfu})$ swab or catheter tip but with negative or no blood culture (see CDC VASC definition for full criteria) ${ }^{18}$ confirmed by a blinded infectious disease specialist.

\section{Secondary outcomes}

- All-cause PICC complication: a composite of the thrombotic and infective complications previously, but evident at any stage during PICC dwell, and may or may not require PICC removal.

- Thrombotic complication: a composite of venous thrombosis, occlusion and breakage at any stage during PICC dwell.

- Infective complication: a composite of PICC-associated BSI and local infection at any stage during PICC dwell.

- Individual complications: individual thrombotic (venous thrombosis, breakage and occlusion) and infective (PICC-associated BSI and local infection) complications evident at any stage during PICC dwell and may or may not require PICC removal.

- Adverse events: any local or systemic allergic reaction (eg, pruritus), pain and mortality. 
- PICC dwell time: hours from PICG insertion until removal.

- Patient/parent and staff satisfaction: collected at PICC insertion (staff) and study end (staff and patient/ parents), using 0-10 numeric rating scales of increasing satisfaction. ${ }^{13}$

- Healthcare costs: estimate of direct product costs, healthcare resource utilisation (including additional equipment and staff time) and failure-associated resource usage (described further). ${ }^{19}$

\section{Sample size and study power}

Our baseline PICC failure rate associated with the conventional PICCs is $15 \% .^{20-22}$ We expect PICC failure of $7.5 \%$ with hydrophobic and CHG PICCs based on pilot trials $^{8}$ and systematic reviews. ${ }^{923}$ That is, we expect hydrophobic and CHG PICCs to have a $50 \%$ relative risk reduction in PICG failure rates compared with conventional PICCs. Using a two-sided alpha significance level of 0.035 (chosen to facilitate comparison of the two intervention arms against a single control arm), 366 PICCs in each group achieves $85 \%$ power to detect a relative $50 \%$ difference in failure rates between intervention ((1) and (2)) hydrophobic and CHG PICCs) and control ((3) conventional PICCs) groups. Consequently, we are required to enrol 1098 participants.

\section{Recruitment, randomisation, allocation concealment and blinding}

Hospital-based research nurses (ReNs) are screening patients, gaining informed consent from patients/ parents, randomising, educating clinical staff, patients and families, monitoring protocol compliance and collecting weekly data on patients 'on trial' plus final outcome data for completed patients. Patients are randomised via a central web-based service (Griffith University) immediately prior to each PICC insertion to ensure allocation concealment until study entry. Randomisation is at in a 1:1:1 ratio between groups, with randomly varied block sizes (up to 20) and stratification by: (1) hospital and (2) hypercoagulable state (adults: high risk of thrombosis as per Michigan Risk Score ${ }^{24}$; paediatrics: previous thrombosis or active cancer).

PICC inserters have been provided training by the product manufacturers regarding the insertion of intervention PICCs, with additional resources available to support practice. The inserters are provided with the randomised PICC in prepacks, immediately prior to insertion, to ensure study fidelity. It is not possible to blind clinical staff, since they must insert, access and monitor PICCs, and they are visibly different. However, the outcome of PICC failure is objective, easily assessed and routinely assigned by clinical staff who are not investigators. Infectious disease physicians and radiologists are masked when determining infection and thrombosis outcomes, standardised definitions are used for these outcomes and the biostatistician will be masked to allocation when provided the dataset.

\section{Insertion and care of PICC}

Other than PICC type, all other PICC insertion, care and management have been standardised in accordance with Queensland Health clinical practice guidelines (at all study sites). ${ }^{25}$ This includes skin preparation with $2 \%$ CHG in $70 \%$ alcohol prior to insertion, sterile insertion, ultrasound guidance for insertion, securement with sutureless securement devices and polyurethane dressings and removal as soon as clinically appropriate. Maintenance and removal will be by usual hospital staff, with existing guidelines in place. As a pragmatic trial, variations in such care are being noted but not considered as protocol violations. A protocol violation is the insertion of a non-randomised PICC.

\section{Data collection}

Data are being directly collected into a secure online database (Research Electronic Data CAPture, Vanderbilt). ${ }^{26}$ The ReNs are following participants weekly for a maximum of 8 weeks (captures $90 \%$ of dwell data; will be censored at that point) or until PICC failure/removal if earlier. Discharged patients are having follow-up data collected by phone, a method that we have previously demonstrated reliability. ${ }^{27}$ The study manager are undertaking quality checks and monitoring $100 \%$ source data verification for: all data for the first patient per site, all consent forms, all primary endpoints and a random $5 \%$ of other data for all patients.

At enrolment, ReNs are collecting consent forms and data on patient, provider and device factors that potentiate PICC-associated complications and failure. ${ }^{2}$ These include: patient (eg, critical illness factors, coagulopathy, diagnostic group, neutropaenia, previous history of CABSI or venous thrombosis); device (PICC lumens, PICC size and vessel size); and provider (eg, inserter discipline, care during insertion and care during access) factors.

Immediately or within 24 hours of recruitment, PICC inserters are asked to rate the ease of PICC insertion ( $0=$ very difficult, $10=$ very easy).

Weekly, the ReNs are visiting or contacting participants to assess for primary and secondary outcomes, current use, and any clinical, PICC or treatment factors that have changed since recruitment.

Within 24 hours of PICC removal or at 8 weeks, the ReNs are collecting data on reason for removal including presence and type of failure if present, PICC dwell and infusates. Also within 24 hours, ReNs are asking the patient/parent about satisfaction with the PICCs $(0=$ completely dissatisfied, $10=$ completely satisfied).

Within 1 week of PICC removal or at 8 weeks (maximum data collection), ReNs are collecting microbiological and clinical data for infectious, thrombotic and microbiological outcomes.

\section{Microbiological substudy}

To determine the effect of (1) hydrophobic and (2) CHG PICCs on bacterial colonisation, PICC-associated infection 
and CHG resistance, compared with (3) conventional PICCs, catheter tips are being sent for further microbiological analysis in a selection of participants following PICC removal. This includes all PICC tips removed due to suspected infection, plus a control group of PICC tips from participants with no symptoms of infection (matched according to randomisation arm at a ratio of 2:1 cases to controls). All PICC tips are being cultured using the roll-plating method on blood agar, with quantification of cfus, species identification using Vitek-MS (BioMerieux) and susceptibility testing for a standard panel of antibiotics by Vitek 2 automated broth microdilution. In addition, CHG tolerance is being tested by determining minimum inhibitory concentrations against organisms isolated from PICC tips using broth microdilution (with an MIC $\geq 4 \mathrm{mg} / \mathrm{L}$ defining reduced susceptibility to $\mathrm{CHG}) .^{28}$ Genes known to be associated with $\mathrm{CHG}$ tolerance (eg, qacA/Band smr) are being confirmed by PCR. In patients with suspected CASBI and the same species grown in PICC tip cultures, organisms isolated from both blood and tip cultures are having DNA extracted and whole genome sequencing performed on the Illumina MiniSeq platform. Raw reads are being trimmed, checked for quality metrics, assembled and analysed using a custom pipeline to confirm species identification, define in silico multilocus sequence type and detect the presence of any antibiotic resistance genes. Other genes known to be associated with CHG tolerance and biofilm formation are being sought by BLAST against the assembled genomes. To confirm whether PICC tip and blood culture isolates are clonal, core genome differences between blood and PICC tip isolates are being determined by comparing single nucleotide polymorphisms.

\section{Thrombogenic substudy}

A subgroup of participants $(n=120)$ are having blood collected on PICC insertion and, if possible, at the time of removal to assess haematological, coagulation profile and platelet activation. Blood samples are being collected via the PICC (from discard and whole blood aspirate) by ReN in sodium citrate and EDTA vacutainers (platelet/ full blood count), and the samples will be analysed for full blood examination (including white blood cell differentiation), clotting times (prothrombin time and activated partial thromboplastin time), platelet activation studies (flow cytometry) and D-dimer. These analyses will link the theoretical proposed mechanism of action to the clinically reported outcomes, strengthening study outcomes. Scanning electron microscopy will semiquantitatively assess blood component adherence to internal and external surfaces of removed PICCs.

\section{Data analysis}

Analysis will be 'intention to treat' with patient the unit of analysis. Baseline characteristics for each of the PICC groups will be descriptively presented using frequencies and percentages for categorical variables and means and SDs (or median and IQR if appropriate) for continuous variables. Primary analyses will compare between-group device failure rates using Cox proportional hazards regression, with treatment group included as the main effect. Effect estimates will be presented for hydrophobic PICCs versus conventional polyurethane PICCs and for CHG-impregnated PICCs versus conventional polyurethane PICCS as HRs with corresponding 95\% CIs. Time-to-event data will be displayed graphically using Kaplan-Meier curves, and the differences between each of the hydrophobic and CHG PICC treatment groups and the conventional polyurethane PICC group will be compared using the log-rank test. The cause of any missing data will be assessed, and sensitivity analyses to investigate the potential impact of missingness will be undertaken using multiple imputation techniques if appropriate. A per-protocol analysis will assess the effect of protocol violations (ie, insertion of an unallocated PICC). $\mathrm{P}$ values $\leq 0.05$ will be considered statistically significant, except for the primary outcome $(\leq 0.031)$, which has been chosen due to the two intervention arms being tested against a single control and to account for the two interim analyses of each intervention arm against control.

\section{Estimating cost parameters}

We hypothesise significantly reduced costs over control from a direct hospital perspective for the episode of care (2020 standardised \$). We will quantify additional costs, cost offsets and net monetary benefit, considering PICC failure, reinsertions and treatment costs of complications per group. A microcosting or bottom-up approach will be used with detailed resource use for PICC insertion/removal recorded for 75 procedures selected at random (25/group). Staff wage costs for application, troubleshooting, replacement, consultation and equipment used will be recorded. Regression analysis of the total cost per patient as the dependent variable will be conducted using a generalised linear model with trial group allocation (either intervention or usual care) as the predictive variable and controlling possible confounders. A gamma family, log-link model has been assumed given the typically skewed nature of health cost data but will be further tested for appropriate model specification. To explore potential differences in health-related quality of life and patient experience, participants are being asked to complete both the EuroQol Five Dimension (EQ-5-D) multiattribute utility instrument at insertion and removal (including EQ-5D-Youth for 8-17yearolds) ${ }^{29} 30$ and the Australian Hospital Patient Experience Question Set, developed by the Australian Commission on Safety and Quality in Health Care, ${ }^{31}$ at removal.

\section{Patient and public involvement}

Feedback regarding the acceptability of the intervention products was sought from patients and their families during the pilot studies, which informed the development of this study. The overall results of the study will be communicated to the study participants by sending a plain language summary, and the associated peer-reviewed articles, to the provided email address.

\section{ETHICS AND DISSEMINATION}

This trial is registered with the Australian New Zealand Clinical Trials Registry. The trial has approval from the 
Children's Health Queensland Hospital and Health Service Human Research Ethics Committee (HREC) (HREC/QCHQ/48682) and Griffith University HREC (Ref. No. 2019/094) and is being managed in accordance with the Australian Government National Health and Medical Research Council (NHMRC) National Statement on Ethical Conduct in Human Research ${ }^{32}$ and the Note for Guidance on Good Clinical Practice (CPMP/ ICH-135/95).

A Data Safety Monitoring Committee will review unblinded data and serious adverse events at $n=400$ and $\mathrm{n}=800$ to advise on any recruitment and safety issues they identify and to investigate whether one or more of the intervention arms should be stopped early for efficacy. The intervention arm will stop recruiting if the arm is significantly different to the control arm at $\mathrm{p}<0.002 .{ }^{33}$

Serious adverse events are being monitored and reported to the HRECs, as are protocol modifications. If important protocol amendments are made (eg, changes to eligibility criteria), AJU will update all investigators, HRECs, patient information and consent forms and the trial registry. Clinical trial insurance is held by the university. Written informed consent is being obtained from participants or representatives prior to participation (see online supplemental file 1 for example of the patient consent form). Consent can be withdrawn. Identifying details are being kept confidential via study IDs.

The trial and substudies were written by the investigators and will be published in peer-reviewed journals using appropriate reporting formats (ie, EQUATOR) with authorship consistent with the International Committee of Medical Journal Editors Guidelines. Data will be held at Griffith University.

\section{Trial status}

The trial began on the 8 September 2019. At the time of manuscript submission, 174 patients had been recruited, with a 2-year recruitment period per study site planned.

\section{Data statement}

The data generated and/or analysed during the trial are not yet publicly available as the trial is ongoing. When the trial is complete, data sets will be available from the chief investigator (AJU) on reasonable request and after agreement by ethics.

\section{DISCUSSION}

PICCs are a common medical device and an important component of modern healthcare, but the current failure rate of up to $30 \%$ is unacceptable and leads patients to experience multiple adverse events. As demonstrated in other vascular access types, innovations in PICC materials and design are an opportunity to reduce this preventable harm to patients and improve efficiencies in healthcare. These technologies are novel and potentially life-saving; however, this independent, sufficiently powered RCT is necessary to ascertain efficacy. Adoption of new technologies without evidence, including economic, may increase expenditure without increased value or cause unintended adverse events.

\section{Author affiliations}

${ }^{1}$ School of Nursing, Midwifery and Social Work, The University of Queensland, St Lucia, Queensland, Australia

${ }^{2}$ Menzies Health Institute Queensland, Griffith University, Nathan, Queensland, Australia

${ }^{3}$ Queensland Children's Hospital, Children's Health Queensland Hospital and Health Service, Brisbane, Queensland, Australia

${ }^{4}$ Royal Brisbane and Woman's Hospital, Metro North Health Service District, Herston, Queensland, Australia

${ }^{5}$ Princess Alexandra Hospital, Metro South Health Service District, Woolloongabba, Queensland, Australia

${ }^{6}$ Faculty of Medicine, The University of Queensland, Saint Lucia, Queensland, Australia

${ }^{7}$ Department of Medicine, University of Michigan, Ann Arbor, Michigan, USA

Twitter Amanda J Ullman @a_ullman, Jessica A Schults @jess_schults and Claire M Rickard @claire_avatar

Acknowledgements We would like to acknowledge the contributions made to the original proposal design by Professors Marianne Wallis and Adrian Esterman. We would also like to acknowledge and thank the clinicians and researchers at each of the study sites.

Contributors AJU, TK, NMM, AB and PNAH conceived the study; AJU and JB designed the economic evaluation; AJU, DA, TK, RW, NMM, RSW, VC, CMR and PNAH designed the protocol; AJU,TK, RW, NMM, AB, BP, NR, PL-A, AC, JB, RW, VC, AC, PM, CMR and PNAH secured funding; all authors prepared and approved the final version of the manuscript.

Funding This work is supported by the National Health and Medical Research Council APP1157178.

Disclaimer No commercial entity had any role in the conception, design or funding of this study, or is the preparation of this manuscript.

Competing interests Griffith University (AJU's former employer) has received unrestricted research and educational grants to support her research (unrelated from the current project) from 3M, Becton Dickinson and Cardinal Health. Griffith University (DA's employer) has received consultancy payments for educational lectures based on her research and clinical expertise from 3M and Johnsons and Johnsons. Griffith University (TK's employer) has received unrestricted research and educational grants from 3M, Becton Dickinson and Medical Specialties Australasia. Griffith University (RMW's employer) has received unrestricted research and educational grants to support her research (unrelated to current project) from Becton Dickinson. Griffith University (NMM's former employer) has received unrestricted research and educational grants to support her research (unrelated from the current project) from 3M, Becton Dickinson and Cardinal Health. Griffith University (AB's employer) has received consultancy payments from Becton Dickinson (unrelated to the current project). Griffith University (JB's employer) has received unrestricted research and educational grants to support her research (unrelated to current project) from Becton Dickinson, and Navi Technologies. PM is a member of Myeloma Advisory Boards for Celgene, Janssen, Amgen, Takeda and Amyloidosis Advisory Boards for Pfizer and Caelum. No personal fees have been received for these services. PM's has received a research grant from Janssen for a clinical trial. PM has accommodation and registration provided by Amgen for attendance at the International Myeloma Workshop in 2019. Griffith University (CMR's former employer) has received unrestricted research and educational grants to support her research (unrelated to the current project) from 3M, Becton Dickinson and Cardinal Health. Other authors have no conflicts of interests to disclose.

Patient and public involvement statement Feedback regarding the acceptability of the intervention products was sought from patients and their families during the pilot studies, which informed the development of this study. The overall results of the study will be communicated to the study participants by sending a plain language summary, and the associated peer-reviewed articles, to the provided email address.

Patient consent for publication Not required. 
Ethics approval Queensland Health (HREC/ QCHQ/48682 and Griffith University (Ref. No. 2019/094).

Provenance and peer review Not commissioned; externally peer reviewed.

Data availability statement The data generated and/or analysed during the trial are not yet publicly available as the trial is ongoing. When the trial is complete, data sets will be available from the chief investigator (AJU) on reasonable request, and after agreement by ethics.

Supplemental material This content has been supplied by the author(s). It has not been vetted by BMJ Publishing Group Limited (BMJ) and may not have been peer-reviewed. Any opinions or recommendations discussed are solely those of the author(s) and are not endorsed by BMJ. BMJ disclaims all liability and responsibility arising from any reliance placed on the content. Where the content includes any translated material, BMJ does not warrant the accuracy and reliability of the translations (including but not limited to local regulations, clinical guidelines, terminology, drug names and drug dosages), and is not responsible for any error and/or omissions arising from translation and adaptation or otherwise.

Open access This is an open access article distributed in accordance with the Creative Commons Attribution Non Commercial (CC BY-NC 4.0) license, which permits others to distribute, remix, adapt, build upon this work non-commercially, and license their derivative works on different terms, provided the original work is properly cited, appropriate credit is given, any changes made indicated, and the use is non-commercial. See: http://creativecommons.org/licenses/by-nc/4.0/.

\section{ORCID iDs}

Amanda J Ullman http://orcid.org/0000-0001-8860-5319

Jessica A Schults http://orcid.org/0000-0002-5406-9519

Robert S Ware http://orcid.org/0000-0002-6129-6736

\section{REFERENCES}

1 Cotogni P, Pittiruti M. Focus on peripherally inserted central catheters in critically ill patients. World J Crit Care Med 2014;3:80-94.

2 Chopra V, Anand S, Krein SL, et al. Bloodstream infection, venous thrombosis, and peripherally inserted central catheters: reappraising the evidence. Am J Med 2012;125:733-41.

3 Chopra V, Anand S, Hickner A, et al. Risk of venous thromboembolism associated with peripherally inserted central catheters: a systematic review and meta-analysis. Lancet 2013;382:311-25.

4 Chopra V, O'Horo JC, Rogers MAM, et al. The risk of bloodstream infection associated with peripherally inserted central catheters compared with central venous catheters in adults: a systematic review and meta-analysis. Infect Control Hosp Epidemiol 2013;34:908-18.

5 Ullman AJ, Marsh N, Mihala G, et al. Complications of central venous access devices: a systematic review. Pediatrics 2015;136:e1331-44.

6 Ullman AJ, Bulmer AC, Dargaville TR, et al. Antithrombogenic peripherally inserted central catheters: overview of efficacy and safety. Expert Rev Med Devices 2019;16:25-33.

7 Witmeyer R, Sukavaneshvar S. Endexo ${ }^{\mathrm{TM}}$ non heparinized surface reduces platelet adhesion and increases catheter lumen patency salt lake City. 2008. Utah: Interface Biologics, 2017.

8 Kleidon T, Ullman AJ, Zhang L, et al. How does your PICCOMPARE? A pilot randomized controlled trial comparing various PICC materials in pediatrics. $J$ Hosp Med 2018;13:E1-9.

9 Lai NM, Chaiyakunapruk N, Lai NA, et al. Catheter impregnation, coating or bonding for reducing central venous catheter-related infections in adults. Cochrane Database Syst Rev 2016;3:CD007878.

10 Ullman AJ, Cooke ML, Mitchell M, et al. Dressing and securement for central venous access devices (CVADs): a Cochrane systematic review. Int J Nurs Stud 2016;59:177-96.

11 Ryder M, Gunther R, Sylvia C. The effect of chlorhexidine catheter coating compared to a biomimetic atheter on the reduction of fibrin sheath formation in an in vivo clinically simulated ovine model. North Carolina, 2016.

12 Pittiruti M, Emoli A, Porta P, et al. A prospective, randomized comparison of three different types of valved and non- valved peripherally inserted central catheters. $J$ Vasc Access 2014;15:519-23.

13 Ullman AJ, Kleidon T, Gibson V, et al. Central venous access device SeCurement and dressing effectiveness (cascade) in paediatrics: protocol for pilot randomised controlled trials. BMJ Open 2016;6:e011197.

14 Lobo BL, Vaidean G, Broyles J, et al. Risk of venous thromboembolism in hospitalized patients with peripherally inserted central catheters. J Hosp Med 2009;4:417-22.

15 Matsuzaki A, Suminoe A, Koga Y, et al. Long-term use of peripherally inserted central venous catheters for cancer chemotherapy in children. Support Care Cancer 2006;14:153-60.

16 Yamamoto AJ, Solomon JA, Soulen MC, et al. Sutureless securement device reduces complications of peripherally inserted central venous catheters. J Vasc Interv Radiol 2002;13:77-81.

17 Goossens GA, De Waele Y, Jérôme M, et al. Diagnostic accuracy of the catheter injection and aspiration (CINAS) classification for assessing the function of totally implantable venous access devices. Support Care Cancer 2016;24:755-61.

18 Centers for Disease Control and Prevention. National healthcare safety network (NHSN) patient safety component manual: bloodstream infection event. in: US Government. Atlanta, 2020: 1-9.

19 Tuffaha HW, Rickard CM, Webster J, et al. Cost-effectiveness analysis of clinically indicated versus routine replacement of peripheral intravenous catheters. Appl Health Econ Health Policy 2014;12:51-8.

20 Rickard CM, Marsh NM, Webster J, et al. Peripherally inserted central catheter dressing and securement in patients with cancer: the Pisces trial. protocol for a $2 \times 2$ factorial, superiority randomised controlled trial. BMJ Open 2017;7:e015291.

21 Rickard CM, Marsh NM, Webster J, et al. Intravascular device administration sets: replacement after standard versus prolonged use in hospitalised patients--a study protocol for a randomised controlled trial (The RSVP Trial). BMJ Open 2015;5:e007257.

22 Kleidon TM, Rickard CM, Schults JA, et al. Development of a paediatric central venous access device database: a retrospective cohort study of practice evolution and risk factors for device failure. $J$ Paediatr Child Health 2020;56:289-97.

23 Kramer RD, Rogers MAM, Conte M, et al. Are antimicrobial peripherally inserted central catheters associated with reduction in central line-associated bloodstream infection? A systematic review and meta-analysis. Am J Infect Control 2017;45:108-14.

24 Chopra V, Kaatz S, Conlon A, et al. The Michigan risk score to predict peripherally inserted central catheter-associated thrombosis. J Thromb Haemost 2017;15:1951-62.

25 Children's Health Queensland Hospital and Services. Venous access device (VAD)-insertion and management of peripheral and central venous access devices. Brisbane: Queensland Government, 2018.

26 Harris PA, Taylor R, Thielke R, et al. Research electronic data capture (REDCap)-A metadata-driven methodology and workflow process for providing translational research informatics support. J Biomed Inform 2009;42:377-81.

27 Webster J, Northfield S, Marsh N. Excellent inter-rater agreement of peripherally inserted central catheter site condition between nurses and patients: PICC-SAT. J Vasc Access 2018.

28 Horner C, Mawer D, Wilcox M. Reduced susceptibility to chlorhexidine in staphylococci: is it increasing and does it matter? $J$ Antimicrob Chemother 2012;67:2547-59.

29 Wille N, Badia X, Bonsel G, et al. Development of the EQ-5D-Y: a child-friendly version of the EQ-5D. Qual Life Res 2010;19:875-86.

30 Herdman M, Gudex C, Lloyd A, et al. Development and preliminary testing of the new five-level version of EQ-5D (EQ-5D-5L). Qual Life Res 2011;20:1727-36.

31 Australian Commission on Safety and Quality in Health Care. Australian hospital patient experience question set. Canberra, Australia: Australian Commission on Safety and Quality in Health Care, 2020.

32 National Health and Medical Research Council. National statement on ethical conduct in human research: 2007 (updated 2018): Commonw. Aust. Canberra, 2018.

33 Freidlin B, Korn EL, Gray R, et al. Multi-Arm clinical trials of new agents: some design considerations: table 1. Clin Cancer Res 2008;14:4368-71. 